\title{
LOCALIZED ACTIVATION OF BENDING IN PROXIMAL, MEDIAL AND DISTAL REGIONS OF SEA-URCHIN SPERM FLAGELLA
}

\author{
C. J. BROKAW* AND I. R. GIBBONS \\ Pacific Biomedical Research Center, University of Hawaii, Honolulu, Hawaii
}

\section{SUMMARY}

Spermatozoa from the sea urchin, Colobocentrotus atratus, were partially demembranated by extraction with solutions containing Triton $\mathrm{X}-100$ at a concentration which was insufficient to solubilize the membranes completely. The resulting suspension was a mixture containing some spermatozoa in which a proximal, medial, or distal portion of the flagellum was membranecovered, while the remaining portion was naked axoneme. In reactivating solutions containing $12 \mu_{\mathrm{M}}$ ATP, only the naked portions of the flagellum became motile. In reactivating solutions containing $0.8 \mathrm{~mm}$ ADP, the membrane-covered regions became motile and beat at 6 -1 0 beats/s, while the naked regions remained immobile, or beat very slowly at about $0.3 \mathrm{beat} / \mathrm{s}$. Activation of membrane-covered regions in ADP solutions probably results from the membrane restricting the diffusion of ATP which is formed from ADP by the axonemal adenylate kinase. The results indicate that any region of the flagellum has the capacity for autonomous beating, and that special properties of the basal end of the flagellum are not required for bend initiation. However, the beating of different regions of the flagellum is not completely independent, for in a fair number of spermatozoa the beating of the distal, membrane-covered region in $0.8 \mathrm{mM}$ ADP was intermittent, and was turned on and off in phase with the much slower bending cycle in the proximal region of naked axoneme.

\section{INTRODUCTION}

Two types of theory have been developed to account for flagellar bend propagation. One type suggests that bending waves will be spontaneously initiated as a consequence of distributed properties of active elements along the length of the flagellum (Machin, 1958, 1963; Miles \& Holwill, 1971 ; Brokaw, 1971, 1972). The second type of theory assumes that bending waves are initiated by a 'control centre' located at the base of the flagellum (Brokaw, I966; Lubliner \& Blum, 197 $a, b$; Rikmenspoel, 197r). Results from experiments with amputated sea-urchin sperm flagella (Goldstein, 1969) have been interpreted as support for the second type of theory, but similar experiments with other flagella appear to support the first (cf. Goldstein, Holwill \& Silvester, 1970; Lindemann \& Rikmenspoel, 1972a,b). In this paper we describe experiments involving the reactivation of partially demembranated sea-urchin spermatozoa in solutions containing ADP or ATP. Our results provide direct support for the first type of theory, and help to explain the conflicting conclusions obtained from other types of experiments.

- Permanent address, to which requests for reprints should be addressed: Division of Biology, California Institute of Technology, Pasadena, California 9 r rog, U.S.A. 


\section{MATERIALS AND METHODS}

Semen was obtained from the sea urchin Colobocentrotus atratus, and was diluted with sea water as necessary to make a sperm suspension containing $10 \mathrm{mg}$ protein/ml (Gibbons \& Gibbons, 1972). Fully Triton-extracted spermatozoa were prepared by adding $25 \mu \mathrm{l}$ of sperm suspension to $0.3 \mathrm{ml}$ of extracting solution containing $0.02 \%(\mathrm{w} / \mathrm{v})$ Triton $\mathrm{X}-\mathrm{1} \infty 0,0.15 \mathrm{M} \mathrm{KCl}$, $4 \mathrm{mM} \mathrm{MgSO}_{4}, 0.5 \mathrm{~mm}$ ethylenediaminetetra-acetate (EDTA), I mM dithiothreitol, and $2 \mathrm{~mm}$ tris-hydroxymethylamino-methane ( $\mathrm{Tris}$ )- $\mathrm{HCl}$ buffer, $\mathrm{pH} 8 \cdot 0$, and mixing gently for $30 \mathrm{~s}$ at room temperature. Incompletely Triton-extracted spermatozoa were prepared in the same way except that the Triton concentration in the extracting solution was lowered to $0.015 \%$, and sometimes the exact extent of extraction was adjusted by varying the relative proportions of sperm suspension and extracting solution. Small volumes of the extracted sperm suspensions were diluted into reactivating solution containing $0.15 \mathrm{M} \mathrm{KCl}, 2 \mathrm{mM} \mathrm{MgSO}_{4}, 0.5 \mathrm{~mm}$ EDTA, I mM dithiothreitol, $2 \%$ polyethylene glycol (mol. wt. 20000), $20 \mathrm{mM}$ Tris-HCl buffer, $\mathrm{pH} 8.0$ and either $12 \mu \mathrm{M}$ adenosine triphosphate (ATP) or $0.8 \mathrm{~mm}$ adenosine diphosphate (ADP), in a flatbottomed glass dish, at $25{ }^{\circ} \mathrm{C}$. The movements of the reactivated sperm were studied by light microscopy, using a ro $x$ objective, with dark-field illumination from a Xenon flash unit (Chadwick-Helmuth Company, Monrovia, California). Cine films were taken at $12-16$ frames per s, with a Sage-Bolex microcinematography apparatus, Model 201. Still photographs were made on Polaroid Type 107 film at a magnification of $425 \times$.

Assays of adenylate kinase activity were performed by incubation in a medium containing $0.8 \mathrm{~mm}$ ADP, $0.15 \mathrm{M} \mathrm{KCl}, 2 \mathrm{~mm} \mathrm{MgSO}, 0.5 \mathrm{~mm}$ EDTA, and $30 \mathrm{~mm}$ Tris-HCl buffer, $\mathrm{pH} 8.0$, at $25^{\circ} \mathrm{C}$. The rate of ATP formation was determined by the coupled-enzyme procedure described previously (Gibbons, 1966), except that in some cases, when assaying adenylate kinase bound to the sperm, the reaction was terminated by centrifugation of the sperm prior to addition of the ATP assay solution.

\section{RESULTS}

\section{Spermatozoa fully extracted with Triton}

The flagellum of a Triton-extracted spermatozoon appears to scatter less light than the flagellum of a live spermatozoon, when observed with dark-field light microscopy. This decrease in the apparent brightness of the flagellum is largely a result of the solubilization of the flagellar membrane, for examination of extracted spermatozoa by electron microscopy has demonstrated that this membrane is completely removed by extraction with Triton concentrations of $0.02 \%$ or higher (Gibbons \& Gibbons, 1972). The movements of the extracted spermatozoa can be reactivated when they are diluted into solutions containing ATP, and the ATP-reactivated movement is very similar to the movement of live spermatozoa (Gibbons \& Gibbons, 1972).

Movements of Triton-extracted spermatozoa can also be reactivated by solutions containing ADP (Fig. 2). With very dilute sperm suspensions in reactivating solution containing $0.8 \mathrm{~mm}$ ADP, bending waves were initiated with frequencies of approximately 0.3 beats per $s$, and were propagated regularly along the flagellum to produce a waveform similar to that which would be characteristic of the movement at much higher frequency (approximately 30 beats per s) in solutions containing $0.8 \mathrm{~mm} \mathrm{ATP.}$ Regular movement at very low beat frequencies is easily and reproducibly obtained in ADP solutions, whereas it is usually difficult to obtain uniform movements at frequencies below about $\mathrm{I}$ beat per $\mathrm{s}$ when the sperm are reactivated with appropriately low concentrations of ATP.

In denser sperm suspensions, the beat frequency of ADP-reactivated movement 
increases with time to somewhat higher values, but always remains below the frequency observed with an equal concentration of ATP. We did not make a detailed study of the factors which determine the beat frequency of ADP-reactivated movement, but our results appear to be consistent with the hypothesis that ADP-reactivated movement is the result of conversion of ADP to ATP by the adenylate kinase in the flagella. Adenylate kinase activity has been demonstrated previously in cilia and flagella from various organisms (Brokaw, 196r; Gibbons, I966). Mohri (1958) presented evidence suggesting that sea-urchin sperm flagella did not have appreciable adenylate kinase activity, but this is not in accordance with our observations.

\section{Measurement and localization of adenylate kinase activity}

Assay of sperm suspensions extracted with $0.04 \%$ Triton showed an average total adenylate kinase activity of $0.21 \mu \mathrm{mol}$ ATP formed per mg sperm protein per min. Increasing the Triton concentration to $0.2 \%$ to effect a more complete solubilization of the membranes and mitochondria (Gibbons \& Gibbons, 1972) did not cause any significant change in adenylate kinase activity.

When the sperm suspension was centrifuged at $2000 \mathrm{~g}$ for $5 \mathrm{~min}$ to separate the Triton-extracted sperm from the solubilized membranes, mitochondria, and matrix protein, about $80 \%$ of the total adenylate kinase activity was found associated with the extracted sperm and the remaining $20 \%$ was in the Triton-soluble supernatant fraction. The soluble portion of the activity may well have been derived from the mitochondria, for these have been shown to possess adenylate kinase activity in other tissues (Siekevitz \& Potter, 1953). The activity associated with the extracted sperm was further localized by homogenizing them, and then separating the axonemes and sperm heads by differential centrifugation. Assay of the resultant fractions indicated that essentially all of the bound adenylate kinase activity was associated with the flagellar axonemes, which had a specific activity of about $\mathrm{I} \cdot 7 \mu \mathrm{mol}$ ATP formed per mg axonemal protein per min, and that there was little or no activity associated with the sperm heads. The rate at which ATP can be formed from ADP under these conditions is equal to or greater than the expected rate of ATP dephosphorylation in a fully motile sea-urchin sperm flagellum (Brokaw \& Benedict, 1971; Gibbons \& Gibbons, 1972).

\section{Spermatozoa incompletely extracted with Triton}

When spermatozoa are extracted with slightly lower Triton concentrations than those which are required for complete removal of the flagellar membrane, the resultant sperm suspensions contain a mixture of spermatozoa with different appearances when observed by light microscopy with dark-field illumination. The flagella of some spermatozoa appear to be completely demembranated, for their brightness is similar to that of sperm extracted with higher concentrations of Triton. These spermatozoa respond to ATP and ADP as described above.

A second class of spermatozoa is found in which the light scattered by the flagellum is significantly greater than that by demembranated flagella, suggesting that the flagellar membrane is still present. Part of the flagellum of these spermatozoa is frequently curled up into a membrane-bounded vesicle, and other distorted con- 
figurations are seen. These spermatozoa show a variety of abnormal beating patterns in reactivating solution, and we have not studied them further.

It is a third class of spermatozoa, found in small and somewhat variable proportion, that is of primary interest. These spermatozoa appear by dark-field microscopy to have a membrane covering one part of the flagellum, while the remaining portion of the flagellum appears to be naked axoneme. The membrane-covered portion was most commonly at the proximal end of the sperm flagellum, adjacent to the head. Less commonly, it was at the distal end of the sperm flagellum, and occasionally, it was located medially, with a region of naked axoneme on either side. The length of the membrane-covered region varied from about $30-70 \%$ of the flagellar length, as judged visually, in most of the spermatozoa; in a few cases, shorter membrane-covered regions were observed. When the membrane-covered region was located medially, it was always quite short (10-30\% of the flagellar length).

When these preparations were observed in reactivating solution containing $12 \mu \mathrm{M}$ ATP, the fully demembranated spermatozoa were beating with frequencies of about I beat per s. Activity was found in only a relatively small fraction of the partially demembranated spermatozoa. In these cases, the demembranated region was beating with a frequency of about $I$ beat per $s$, while the membrane-covered region was inactive. In most instances, the active demembranated region was at the proximal end of the flagellum, but in a small number of cases the demembranated region at the distal end was beating while the membrane-covered proximal end was inactive.

Much more satisfactory partial activation was obtained with reactivating solutions containing ADP. Under the conditions we used, the majority of the partially demembranated spermatozoa showed partial activation, with the membrane-covered regions of the flagella beating while the demembranated regions showed little or no activity. The general pattern of partial reactivation observed with ADP was thus complementary to that observed with ATP. In reactivating solutions containing $0.8 \mathrm{~mm}$ ADP, many spermatozoa were observed in which the proximal, membranecovered region of the flagellum was beating at a frequency of $5-6$ beats per $\mathrm{s}$, while the naked, distal region was motionless (Fig. 3). In a very few cases, the demembranated distal region of the flagellum was also beating, but at a much lower frequency (less than $\mathrm{I}$ beat per s) than the proximal region. When the membrane-covered region was located at the distal end of the flagellum of a partially demembranated spermatozoon, its beat frequency was normally 9-Io beats per s, significantly higher than the frequency of proximal membrane-covered regions. Fig. 4 shows an example of this type of movement, where the proximal region of the flagellum is not bending. The bending waves in distal regions characteristically had smaller wavelength and amplitude than the bending waves of fully Triton-extracted sperm flagella (compare with Fig. 2), so that one or more complete waves could be seen in a relatively short length of active flagellum.

In approximately half of the cases where the distal, membrane-covered region was actively beating, the proximal demembranated region was also beating, at a much lower frequency of about 0.3 beats per $\mathrm{s}$, similar to that observed in the fully demembranated spermatozoa. Fig. 5 illustrates this type of movement pattern. In the 

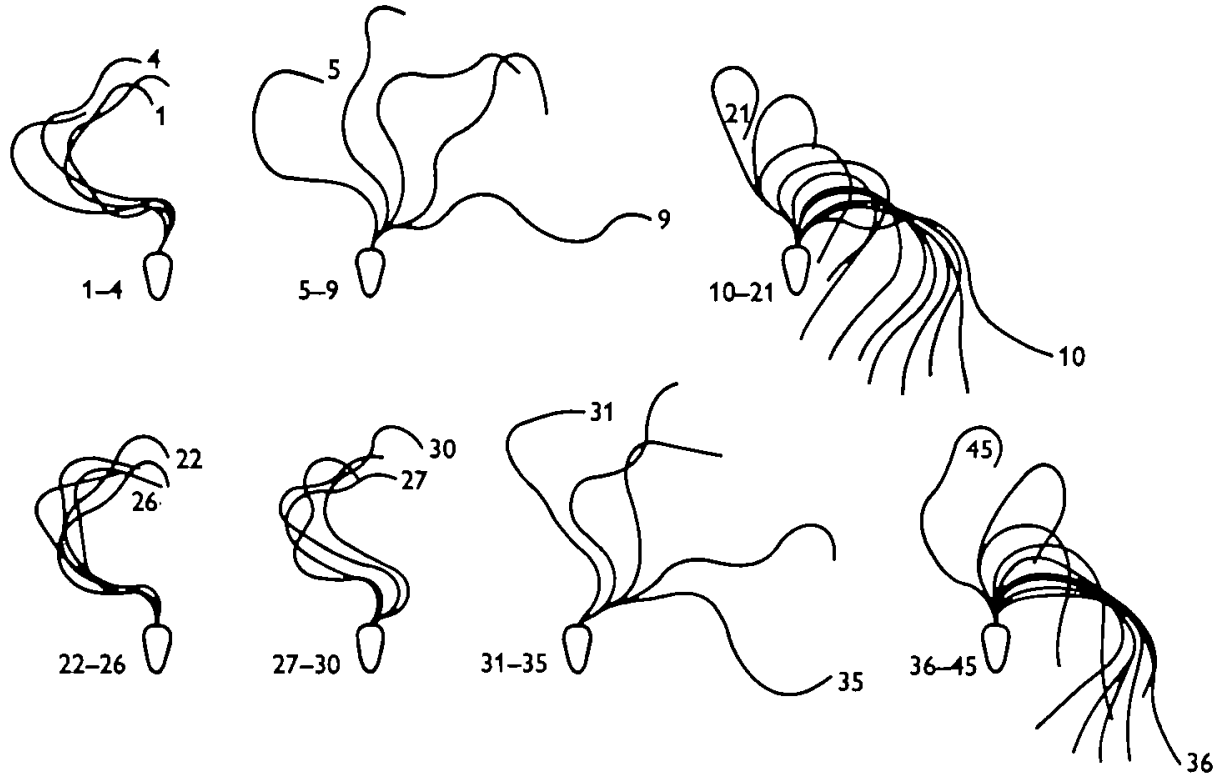

Fig. I. Tracings from a $16-\mathrm{mm}$ film record of an intermittently beating sperm flagellum. The images have been repositioned to eliminate the pivotal motion of the sperm head around its attachment point, in order to emphasize the bending of the flagellum relative to the sperm head. However, it should be remembered that the hydrodynamic conditions experienced by this spermatozoon were not equivalent to the conditions which would have been experienced if the head were not free to pivot.

The distal, membrane-covered portion of the sperm flagellum was about $60 \%$ of the total length of the flagellum and was beating at approximately 8 beats per $s$ during frames $1-9$ and $22-35$. The distal portion was not beating during frames $10-21$ and 36-45. These tracings cover 2 complete cycles of the slow bending in the proximal demembranated region of the flagellum. The correlation shown here between the bending cycle of the proximal region and the activity of the distal region was maintained for at least 10 beat cycles, which were analysed in a similar manner.

majority of spermatozoa which showed this combination of high-frequency beating in the distal, membrane-covered region and low-frequency beating in the proximal, demembranated region, the beating of the distal region was intermittent. There was a regular alternation of periods of beating of the distal region with periods of inactivity, and this cycle was correlated with the beat cycle of the demembranated proximal portion. Fig. I, obtained by tracing a portion of a cine film record of such a spermatozoon, shows the pattern which was typical of the 4 spermatozoa which we recorded and analysed. This record suggests that the high-frequency beating of the distal region is inhibited during the period in which the proximal region is developing a bend towards the left in Fig. I (frames I0-2I and $36-45$ ). During the other half of the beat cycle of the proximal region, the distal region beats with a regular rhythm.

A very few observations were made on flagella in which a medial region of the flagellum was membrane-covered, with demembranated axoneme on either side, as only a few such flagella were obtained. These observations were only made in the presence of $0.8 \mathrm{~mm}$ ADP. When the medial, membrane-covered region was very short (10-20\% of the flagellar length, or of the order of $5 \mu \mathrm{m}$ ), its movement consisted of a 
rapid, rocking, see-saw type motion, in which it appeared to move without noticeable bending while changing its inclination relative to the rest of the flagellum. These movements by the membrane-covered region appeared to be superimposed on the much slower bending waves generated by the remainder of the flagellum. With a slightly longer medial membrane-covered region, this region was also observed to bend, usually into a small 'S-shaped' curve. These movements, and their interaction with the movement of the adjoining demembranated regions of the flagellum appeared to be considerably more complicated, and require much more detailed analysis. Although the movement of a medial membrane-covered region can be clearly seen in our cine films, photographs at higher resolution will be required for detailed analysis, because of the small amplitude of the movements.

\section{DISCUSSION}

A membrane-covered region of the flagellum of a sea-urchin spermatozoon beats more rapidly than a demembranated region when exposed to $0.8 \mathrm{~mm} \mathrm{ADP}$, but does not beat when exposed to low concentrations of ATP. These results are most easily interpreted by assuming that the membrane-covering which can be detected by darkfield microscopy retains some of its ability to restrict the diffusion of ATP between the axoneme and the solution. At low ATP concentrations, the diffusion of ATP through the residual membrane is too slow to allow activation of movement in that region of the flagellum. At the much higher concentrations of ADP used in our experiments, the diffusion of ADP into the membrane-covered region apparently supplies the axoneme with ADP which can be converted to ATP by the axonemal adenylate kinase. The residual membrane allows the ATP to accumulate in higher concentration in the covered regions, than in the uncovered regions where the ATP can diffuse freely away into the bulk of the solution. Our results indicate that the major portion of the flagellar adenylate kinase is associated with the axoneme, and there is no evidence to suggest that the flagellar membrane contains a significant amount of additional adenylate kinase activity.

The beat frequency of the membrane-covered regions will be a complicated function of the membrane permeability to ADP and ATP, the axonemal adenylate kinase and ATPase activities, and the external ADP concentration. The beat frequency of membrane-covered regions located at the distal end of the flagellum is consistently greater than that of similar regions located at the proximal end. Without additional information we cannot say whether this difference in frequency results from the effects of the different physical restrictions on the sliding of the tubules at the proximal and distal ends, or from a variation in amount of adenylate kinase at different locations along the axoneme, or from some other unknown factor. It may be noted that a greater amount of adenylate kinase activity in distal regions of the flagellum might be advantageous to the function of a flagellum which was supplied with ATP by diffusion from the proximal end, but there is no direct evidence to support this hypothesis. A more detailed discussion of the relationship between adenylate kinase activity and ATP diffusion in flagella has been presented by Raff \& Blum ( 1968 ). 
ADP-reactivation of partially demembranated spermatozoa appears to be a convenient procedure for applying ATP to a limited region along the length of a flagellum. Goldstein (1968) attempted to activate glycerinated sea-urchin sperm flagella locally by applying ATP with a micropipette. However, under his conditions the ATP diffused too rapidly to get a sufficiently localized concentration, and he was unable to activate a distal region of the flagellum without also activating the proximal region.

Our results clearly indicate that it is possible for a distal region of a sperm flagellum to beat independently of active movement in the proximal region, and suggest that there is no need for a 'control centre' at the proximal end of the flagellum in order to initiate bending waves. Any portion of the flagellum supplied with ATP can apparently undergo rhythmic activity, and initiate propagated bending waves if it is of sufficient length. This degree of autonomy is consistent with theories for the control of bend propagation proposed by Machin (1958, 1963) and by Brokaw (1971, 1972) which require only a local feed-back between bending and the active process. Our observation that 2 very different beat frequencies can occur simultaneously in different regions of a single axoneme clearly indicates that the beat frequency in a localized region of an axoneme is determined at least in part by the local ATP concentration in that region.

On the other hand, a flagellum normally beats at a uniform frequency over its entire length, even though a substantial diffusion gradient of ATP may exist along the flagellum. Kaneda ( 1965$)$ found, however, that a distal region of a frog sperm flagellum beat at a lower frequency than a proximal region, when these regions were partially isolated by a mechanical obstruction.

Previous experimental work has shown that several kinds of flagella, particularly long sperm flagella from insects and amphibians, can show sustained, rhythmic bending in a distal region of the flagellum after amputation from the proximal end (Terni, 1933; Hoffmann-Berling, 1955; Goldstein et al. 1970; Lindemann \& Rikmenspoel, 1972 $a, b)$. However, with sea-urchin spermatozoa, laser-amputated distal regions stopped initiating bending waves (Goldstein, 1969), and the motility of reactivated sea-urchin spermatozoa can be completely inhibited by breaking the axonemes into pieces by gentle homogenization (Brokaw \& Benedict, I968; Gibbons \& Gibbons, 1972). Our observations on the intermittent beating of the distal region of ADPreactivated spermatozoa possibly help to resolve this conflict, by showing that the movement of the distal region can be modulated by conditions in the proximal region, even though the rhythmic initiation of bending waves is an autonomous feature of the distal region. A somewhat similar effect was noticed by Lindemann \& Rikmenspoel $(1972 a, b)$. They found that regular beating of an isolated distal region of a bull sperm flagellum was obtained only when the piece of flagellum was pushed into a bent position by a microneedle. Their observation suggests that the configuration imposed on the distal region by external influences is a factor which can modulate the activity of the distal region. On the other hand, our results suggest that the direction of bending of the proximal region, which probably correlates with the direction of sliding between filaments in the distal region, can modulate the activity of the distal region. 
Current ideas about mechanisms of flagellar movements are based on the observation that the flagellar tubules whose sliding is responsible for bending are continuous along the whole length of the axoneme (Gibbons \& Grimstone, 1960; Brokaw, I97I ; Summers \& Gibbons, I97I). It seems very reasonable to expect that proximal and distal regions of a flagellum will interact with each other, even under conditions which stimulate autonomous movements in different regions. Further, more detailed study of these interactions may be an important source of information about the control mechanisms involved in normal flagellar bending.

We are grateful to Dr Barbara Gibbons for help in preparing the Triton-extracted spermatozoa, and to Dr John Arnold for the use of his microcinematographic equipment.

This work has been supported in part by grants from the National Institutes of Health (GM I 87 I I, GM I $5090, \mathrm{HD}$ 06565).

\section{REFERENCES}

Brokaw, C. J. (I96I). Movement and nucleoside polyphosphatase activity of isolated flagella from Polytoma uvella. Expl Cell Res. 22, i 5 I-162.

Brokaw, C. J. (1966). Bend propagation along flagella. Nature, Lond. 209, 161-163.

Brokaw, C. J. (1971). Bend propagation by a sliding filament model for flagella. $\mathcal{J}$. exp. Biol. 55, 289-304.

Brokaw, C. J. (1972). Computer simulation of flagellar movement. I. Demonstration of stable bend propagation and bend initiation by the sliding filament model. Biophys. F. 12, 564-586.

Brokaw, C. J. \& Benedict, B. (I968). Mechanochemical coupling in flagella. I. Movementdependent dephosphorylation of ATP by glycerinated spermatozoa. Archs Biochem. Biophys. 125, 770-778.

Brokaw, C. J. \& Benedict, B. (197I). Mechanochemical coupling in flagella. III. Effects of some uncoupling agents on properties of the flagellar ATPase. Archs Biochem. Biophys. 142, $9 \mathrm{r}-\mathrm{r} \infty \mathrm{O}$.

GibBons, I. R. (1966). Studies on the adenosine triphosphatase activity of 145 and 308 dynein from cilia of Tetrahymena. 7. biol. Chem. 24I, 5590- 5596.

Grbions, B. H. \& Gibbons, I. R. (1972). Flagellar movement and adenosine triphosphatase activity in sea-urchin sperm extracted with Triton X-100. F. Cell Biol. 54, 75-97.

Gibbons, I. R. \& Grimstone, A. V. (1960). On flagellar structure in certain flagellates. $\mathcal{F}$. biophys. biochem. Cytol. 7, 697-716.

GoldsteIn, S. F. (I968). Local Activation and Inactivation Experiments on Flagella. Ph.D. Thesis, California Institute of Technology.

GoldsteIN, S. F. (1969). Irradiation of sperm tails by laser microbeam. 7. exp. Biol. 5Y, $43 I-44 I$.

Goldstein, S. F., Holwill, M. E. J. \& Silvester, N. R. (1970). The effects of laser microbeam irradiation of the flagellum of Crithidia (Strigomonas) oncopelti. F. exp. Biol. 53, $40 I-4 \circ 9$.

Hoffman-Berling, H. (I955). Geisselmodelle und Adenosintriphosphat (ATP). Biochim. biophys. Acta I6, I46-I 54 .

Kaneda, Y. (1965). Movement of sperm tail of frog. Y. Fac. Sci. Tokyo Univ., Zool. 10, 427440.

LindemanN, C. B. \& RikmENSPOEL, R. (1972a). Sperm flagella: autonomous oscillations of the contractile system. Science, $N . Y$. 175, 337-338.

LindemanN, C. B. \& Rikmenspoel, R. (1972b). Sperm flagellar motion maintained by ADP. Expl Cell Res. 73, 255-259.

Lubliner, J. \& Blum, J. J. (I97 I $a$ ). Model for bend propagation in flagella. F. theor. Biol. 31, I-24.

LUbliNER, J. \& BLUM, J. J. ( $197 \mathrm{I} b$ ). On the initiation and propagation of bending waves in cilia and flagella. F. Mechanochem. Cell Mot. 1, I 5-22. 
MaChIN, K. E. (I958). Wave propagation along flagella. f. exp. Biol. 35, 796-806.

Machin, K. E. (1963). The control and synchronization of flagellar movement. Proc. R. Soc. B I58, 88-104.

Miles, C. A. \& Holwill, M. E. J. (197I). A mechanochemical model of flagellar activity. Biophys. 7. 11, 851-859.

Mohri, H. (1958). Adenosinetriphosphatases of sea-urchin spermatozoa. F. Fac. Sci. Tokyo Univ., Zool. 8, 307-315.

RafF, E. C. \& Blum, J. J. (1968). A possible role for adenylate kinase in cilia: Concentration profiles in a geometrically constrained dual enzyme system. F. theor. Biol. 18, 53-7r.

Rikmenspoel, R. (1971). Contractile mechanisms in flagella. Biophys. f. II, 446-463.

Siekevitz, P. \& POTTER, V. R. (1953). The adenylate kinase activity of rat liver mitochondria. 7. biol. Chem. 200, 187-1 96.

Summers, K. E. \& Gibbons, I. R. (197I). Adenosine triphosphate-induced sliding of tubules in trypsin-treated flagella of sea-urchin sperm. Proc. natn. Acad. Sci. U.S.A. 68, 3092-3096.

Terni, T. (I933). Microdissection et U. V. microradiopiqure des spermatozoides. C. r. Ass. Anat. 28, $65^{1}-654$.

(Received I9 October 1972) 


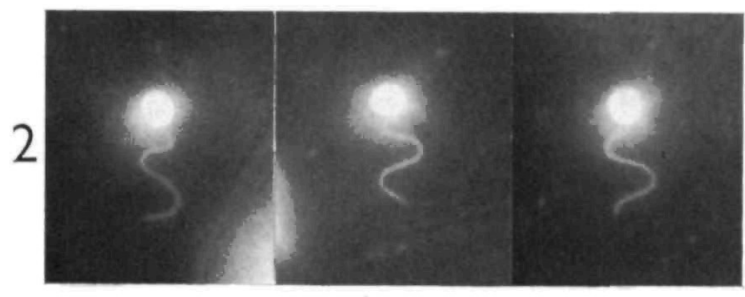

\section{$50 \mu \mathrm{m}$}
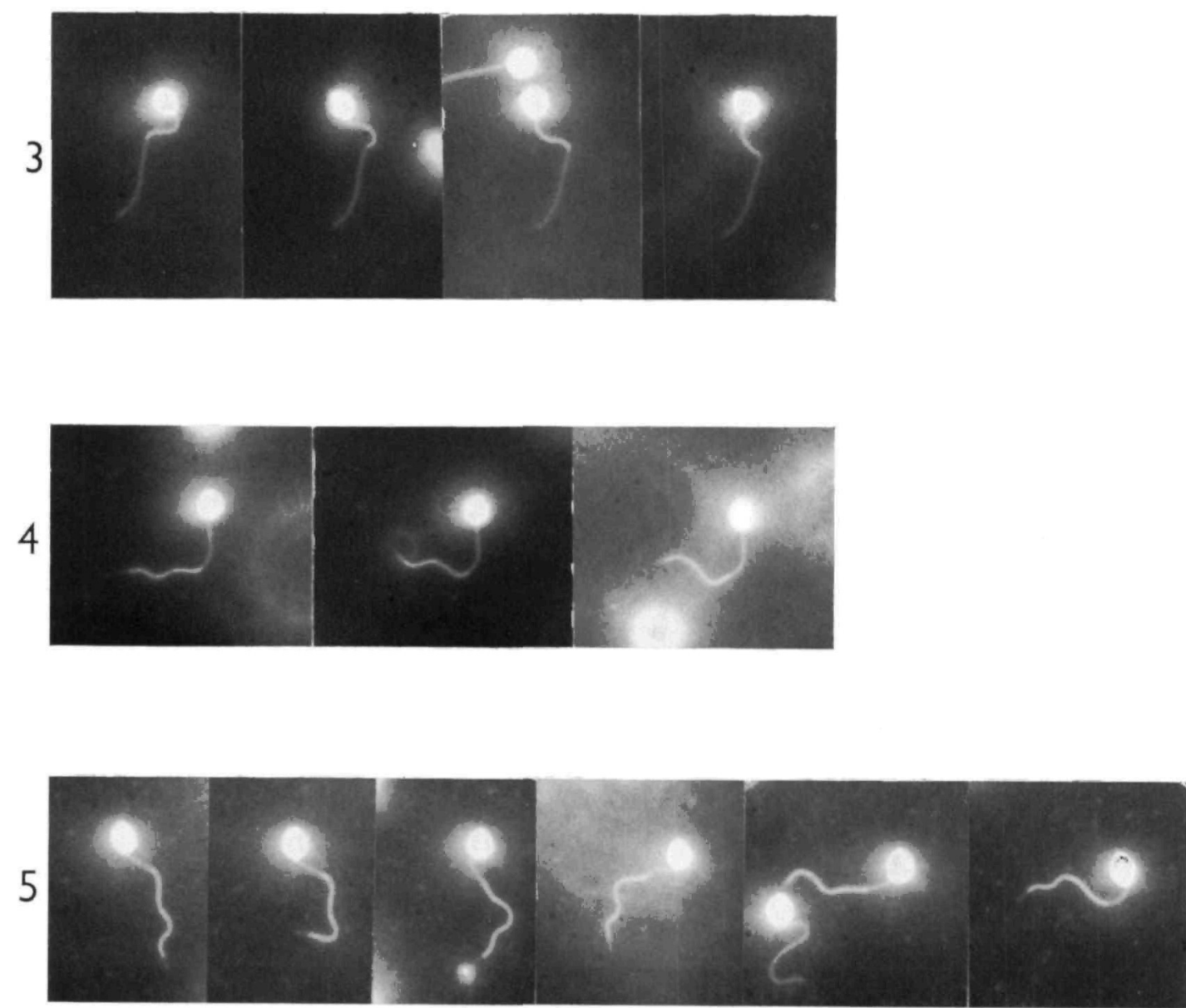

Each figure shows a sperm flagellum in several positions of its beat cycle. The sequences have been synthesized from single exposures, taken at random times relative to the beat cycle, so the photographs do not provide information about the time intervals between successive positions of the flagellum.

Fig. 2 shows a spermatozoon which has been fully extracted with Triton.

Figs. 3-5 show spermatozoa which have been only partially extracted with Triton, so that the flagellar membrane has been removed from only a portion of the length of the flagellum. In Fig. 3, the membrane has been removed from the distal end of the flagellum, while in Figs. 4 and 5, the membrane has been removed from the proximal end of the flagellum.

In Figs. 2-4, the sperm head was attached to the microscope slide by its tip, and the sperm was slowly pivoting around this point of attachment. In Fig. 5, the sperm head was more firmly attached to the slide and was not pivoting around its attachment point. 